\title{
Gut bacterial profile in Indian children of varying nutritional status: a comparative pilot study
}

\author{
Chandrasekhar Balasubramaniam ${ }^{1} \cdot$ Rashmi Hogarehalli Mallappa ${ }^{1} \cdot$ Devendra Kumar Singh ${ }^{1} \cdot$ Pankaj Chaudhary ${ }^{2}$. \\ Bhavneet Bharti ${ }^{2}$. Santhosh Kumar Muniyappa ${ }^{1}$. Sunita Grover ${ }^{1}$
}

Received: 2 February 2021 / Accepted: 19 April 2021 / Published online: 30 April 2021

○) Springer-Verlag GmbH Germany, part of Springer Nature 2021

\begin{abstract}
Purpose Childhood malnutrition is a multifactorial disease, responsible for nearly half of all deaths in children under five. Lately, the probable association of a dysbiotic gut to malnutrition is also being eagerly investigated. The current study is an attempt to investigate this purported association through assessing the abundance of major gut bacterial phyla (Firmicutes, Bacteroidetes, Actinobacteria and Proteobacteria), probionts (Bifidobacteria and Lactobacillus), butyrogens (Faecalibacterium and Roseburia) and pathogens (Escherichia and Klebsiella).

Methods The study was conducted in the suburbs of Chandigarh, India in the year 2017. The children enrolled in the study were part of Anganwadis (Rural Child Care Centres) set up under Integrated Child Development Scheme (ICDS) of Government of India where community-based management approach is being widely used for treatment of malnutrition. We used qPCR based absolute quantification as well as the $16 \mathrm{~S}$ rRNA amplicon sequencing approach for our study. The study population included 30 children in the age group of 2-5 years who were categorized into three groups Healthy, Moderate Acute Malnutrition (MAM) and Severe Acute Malnutrition (SAM), with 10 children in each group. The selection of participants was made based on Z scores. Further, statistical tools like the One-way ANOVA, PCA and PLSDA were employed to analyze and compare the gut bacterial profile.

Results Our investigation through the qPCR (Absolute quantification) approach revealed a significantly higher abundance of Actinobacteria in healthy, in comparison to children suffering from Severe Acute Malnutrition (SAM). Consequently, the same trend was also reflected with respect to Bifidobacterium, a prominent member of the Actinobacteria phylum. Conversely, a significant higher abundance of Lactobacillus with the diminishing nutritional status was recorded. Escherichia showed a significant higher abundance in healthy subjects compared to the malnourished; however, no such difference in abundance of Klebsiella was observed. The other target phyla [Bacteroidetes, Firmicutes and Proteobacteria] and genera (Faecalibacterium and Roseburia) showed differences in abundance; however, these were non-significant. Similarly, the bacterial taxonomy analysis of 16S rRNA gene amplicon sequencing data revealed the higher abundance of phylum Actinobacteria and its member Bifidobacterium with lower prevalence of Lactobacillus in healthy children.

Conclusion The pattern of gut microbiota profile in malnourished subjects suggests a dysbiotic gut depleted in Bifidobacteria, a core member of the consortia of beneficial anaerobes of the healthy child gut.
\end{abstract}

Keywords Gut microbiota $\cdot$ Severe acute malnutrition $\cdot$ Actinobacteria $\cdot$ Bifidobacteria and malnutrition

Chandrasekhar Balasubramaniam and Rashmi Hogarehalli Mallappa contributed equally.

Sunita Grover sungro@gmail.com

1 Molecular Biology Unit, Dairy Microbiology Division, ICAR-National Dairy Research Institute, Karnal, Haryana 132001, India

2 Advanced Paediatrics Centre, Postgraduate Institute of Medical Education and Research, Chandigarh 160012, India

\section{Introduction}

Malnutrition, a nutritional abnormality, has metamorphosed into a pandemic which is notorious for claiming millions of innocent lives worldwide. Malnutrition is prevalent in different forms such as, poor child growth and development; as individuals who are skinny or prone to infection and obesity being the other extreme. Childhood malnutrition is a formidable challenge, threatening the nutritional 
security of children. It is prevalent in all nations in one or the other forms but the underdeveloped and developing countries are the ones which are most affected. India is home to $35 \%$ stunted, $48 \%$ underweight children and another $17 \%$ are prone to wasting [1]. Moreover, malnutrition is also responsible for $69 \%$ of deaths in children below five in India [1]. These alarming figures make India as one of the worst performers in the global malnutrition rankings. Malnutrition has been categorized into different forms i.e., 'Moderate Acute Malnutrition' (MAM) and 'Severe Acute Malnutrition' (SAM) based on Z scores given by World Health Organization (WHO). Malnutrition occurs as a complex interplay of multiple factors like poverty, food insecurity, unhygienic practices, diseases and illiteracy. Further, the interplay of under nutrition and infection triggers a potential lethal cycle, predisposing children to common infections by increasing their frequency of infections and delaying recovery with poor immune status. Of late, an increasing body of facts have hinted at the possible association between the 'gut microbiome' in general ('dysbiotic gut' in particular) and malnutrition [2-7].

The shaping of the 'gut microbiome' starts at the neonatal stage and is influenced by several factors like maternal health and nutritional status of mother, mode of delivery, diet and geographical location. The insights into the gut microbiome have revealed that a healthy intestinal microbiota is quintessential for human health as they perform a wide range of protective, structural and metabolic functions and affect host nutrition and immunity directly or indirectly [8-10]. The commensal bacteria positively modulate the gut functions through several means i.e., through enhancing the intestinal epithelial barrier integrity, providing nutrients to colonic epithelial cells and producing essential vitamins $\left(B_{3}, B_{5}, B_{6}\right.$, $\mathrm{B}_{12}$, biotin, tetrahydrofolate and vitamin K) [10-12]. Further, they also assist in nutrient absorption through inducing specific host genes for nutrient uptake and also through modulating systemic lipid and glucose metabolic rates and gastrointestinal transit time by stimulating and responding to broad range of neurotransmitters [13].

The healthy mature gut microbiota is predominated by an anaerobic microbial consortium which contributes a significant proportion of beneficial microbes to the gut apart from the aerobic ones. These beneficial microbes help to suppress the growth of the pathogenic microbes. In a recent study [14], the significance of the absence of crucial anaerobic microbes in mice, which led to diminished production of $\operatorname{Ig} \mathrm{A}$, an essential defense against pathogenic microbes was elucidated. Further, it is also postulated that these missing microbes that form the core of healthy mature anaerobic gut microbiota (HMAGM), when supplemented in dietetic intervention for malnutrition can rectify the dysbiosis and undo the ill effects of malnutrition $[15,16]$. The ability of beneficial microbes to rectify dysbiosis under malnourished condition has also been experimented with Lactobacillus rahmnosus GG [17] and was found to deliver promising results.

Advances in molecular biology, including 16S rRNA sequencing and polymerase chain reaction, compounded with the developing science of "culturomics" have allowed exploration into gut microbial ecosystem and dynamics. The studies through various sequencing approaches have brought about the differences in the gut microbiota of healthy and malnourished children, which point towards a dysbiotic gut $[6,18]$. The malnourished gut of children has been found to have reduced diversity of microbiota and also has a smaller number of butyrogenic and probiotic bacteria in comparison to their healthy counterpart $[5,6]$. The healthy child gut was dominated by microbes belonging to orders Lactobacillales, Enterobacteriales, Pseudomonadales, Chloroflexales, Xanthomonadales, Planctomycetales, Halobacteriales, Burkholderiales, Actinomycetales, Bifidobacteriales, Desulfovibrionales and Rhizobiales, whereas the malnourished gut was found to harbor more of microbes belonging to the family Campylobacteraceae, Helicobacteracaeae and Bacteriodaceae [2]. This altered gut predominated by pathogenic microbes is believed to confer a reduced immunity by increasing proclivity towards inflammatory diseases through an increased proneness to a leaky gut. The leaky gut predisposes the child to frequent bouts of infections due to villous atrophy, anorexia and finally ending up in impaired growth leading to malnutrition [19]. It has been proposed that the dysfunctional communities of gut microbes conspire with the poor diet to bring about malnutrition [13].

Though few studies have been conducted on similar lines in India, our study is one of the first such study involving children from northern region of India. Additionally, as already mentioned gut microbiota is greatly influenced by geography and other environmental associated factors including diet being a major determinant. India being a diverse country with varied geography, climate and diet spanning the length and breadth of the country, it is bound to have effects on the gut microbiome. Hence, conducting region-specific (Pan-India) studies on gut microbiome can give a better insight and generate corroborative evidences for making meaningful inferences regarding the child gut microbiome. Further this study also involved participants from Anganwadis (Rural Child Care Centres) practicing the community-based management approach for treating malnutrition, which we believe to be one of the only studies conducted till date, enrolling such participants. The current study envisages to assess the association between an altered gut microbiota (depleted in healthy mature anaerobic microbes and the other beneficial ones) and malnutrition through comparative analysis of the gut microbiota profile in healthy and malnourished children (MAM and SAM) by assessing the 
abundance of targeted gut bacterial phyla Bacteriodetes, Firmicutes, Proteobacteria and Actinobacteria, pathobionts (Klebsiella, Escherichia), probionts (Lactobacillus, Bifidobacteria), and butyrogenic bacteria (Faecalibacteria and Roseburia) through qPCR approach and validating the results of the qPCR study with untargeted 16rRNA gene amplicon sequencing.

\section{Methods and materials}

\section{Screening and selection of subjects for the study}

The subjects enrolled in this study included children who fell into the age group of 2-5 years. The study population was selected from "Anganwadis" (Rural Child Care Centres) located in the suburbs of Chandigarh $\left(30.7333^{\circ} \mathrm{N}\right.$, $76.7794^{\circ}$ E) (Union Territory of India) in the year 2017. These anganwadis practiced community-based management approach for treating malnutrition. The subjects were identified and approached with the help of social workers from the "Social Paediatrics" department of the "Advanced Paediatric Centre" (APC) of Post Graduate Institute of Medical Education and Research (PGIMER), Chandigarh. The samples were collected with the consent of the concerned authorities of PGIMER after the study was ethically approved by the Institutional Ethics Committee (IEC) of PGIMER, Chandigarh (Ref.no. NK/2519/MD/538-39). Additionally, an informed consent was also sought from the parents/guardians of the children enrolled in the study. The purpose of the study was explained to the anganwadi workers as well as the donor's parents. The subjects were screened and segregated into the three target groups i.e., healthy, MAM (Moderate Acute Malnutrition) and SAM (Severe Acute Malnutrition) based on $Z$ scores, obtained with WHO ANTHRO $Z$ score calculator software (http://www.who.int/childgrowth/softw are). To derive the $Z$ scores using the software, the height, weight was measured and age of the subjects was recorded. Besides, the inclusion and exclusion criteria for the selection of subjects were laid down prior to the collection of faecal samples. The inclusion criteria included the factors such as children of age between $2-5$ years and standard $Z$ score -2 to $+2,-2$ to -3 and $<-3$ for grouping them into various nutritional health status such as healthy, MAM and SAM respectively. The exclusion criteria included (1) the children suffering from episodes of diarrhoea (2) consumption of antibiotics or probiotics 3 months preceding the date of sample collection Fig. S1.

A total of 30 subjects, with an average age of 3 years which included ten healthy, ten Moderate Acute Malnourished (MAM) and ten Severe Acute Malnourished (SAM) children were selected.

\section{Collection of samples and extraction of metagenomic DNA}

The parents of the selected subjects were provided with the sterile faecal/stool sample collection containers the day before the collection of morning faecal samples and were explained about the procedure to collect the samples. The daily collected samples were recovered from the parents at anganwadis and transported to the Molecular Biology Unit of Dairy Microbiology Division, ICAR-National Dairy Research Institute (ICAR-NDRI) in insulated ice boxes on the same day of collection. All the collected samples were stored at $-80^{\circ} \mathrm{C}$ in the Ultra-Low Deep Freezer (SANYO, Japan) till further processing for metagenomic DNA extraction.

The collected faecal samples were thawed in ice on the day of extraction of metagenomic DNA and the faecal DNA was extracted using Zymo Research Faecal DNA Miniprep kit Claassen et al. [20] as per manufacturer's instructions. The metagenomic DNA extracted above was analyzed for quantity, purity and integrity for its downstream applications like PCR and Real-Time PCR (qPCR) studies. Metagenomic DNA was quantified spectrophotometrically by using Synergy H1 Multi-Mode Reader (BioTek, Winooski, VT), in which Nucleic acid quantification was done at $260 \mathrm{~nm}$. Metagenomic DNA extracted using Zymo Research Mini Prep. Kit was analyzed for its integrity for downstream applications by electrophoresing in $0.8 \%$ agarose gel.

The extracted DNA from all the 30 faecal samples was evaluated for their PCR amenability by $16 \mathrm{~S}$ rRNA gene amplification using universal primers. The PCR cycling conditions for the selected 16S rRNA universal primers included initial denaturation of $95^{\circ} \mathrm{C}$ for $5 \mathrm{~min}$ and 35 cycles each of denaturation $95^{\circ} \mathrm{C} / 30 \mathrm{~s}$, annealing $60{ }^{\circ} \mathrm{C} / 30 \mathrm{~s}$ and extension $72{ }^{\circ} \mathrm{C} / 2 \mathrm{~min}$ with final extension at $72{ }^{\circ} \mathrm{C}$ for $10 \mathrm{~min}$. The amplicons were visualized by electrophoresing the products of the PCR reaction on a 1.8\% agarose gel.

\section{Selection of primers for target bacterial groups and specificity analysis.}

The primers for all the selected gut microbial enterotypes such as Eubacteria, Firmicutes, Bacteroidetes, Proteobacteria, Actinobacteria, Lactobacilli, Bifidobacteria, Roseburia, Faecalibacteria, Escherichia and Klebsiella were selected from the literature as illustrated in Table 1 [21-31]. Initially, the selected primers were evaluated in silico for specificity by BLAST (Basic Local Alignment Search Tool) analysis prior to getting them synthesized. Orders for the suitable primers were placed and got synthesized from Imperial Biomed [Integrated DNA Technologies, Inc]. The specificity of the primers was further evaluated by agarose gel electrophoresis and the 
Table 1 Sequences and respective product sizes of primers used in the absolute quantification of target gut enterotypes used in the study

\begin{tabular}{|c|c|c|c|c|}
\hline Target Phyla/Genera & Primer ID & Sequence & $\begin{array}{l}\text { Product size } \\
\text { (bp) }\end{array}$ & References \\
\hline \multirow[t]{2}{*}{ Eubacteria } & Eub-1F & $5^{\prime}$ act cctacgggaggcagcag $3^{\prime}$ & 200 & {$[26]$} \\
\hline & Eub-1R & $5^{\prime}$ attaccgcggctgctgctgg $3^{\prime}$ & & \\
\hline \multirow[t]{2}{*}{ Bacteroidetes } & Bacphy-1F & $5^{\prime}$ ccggawtyattgggtttaaaggg $3^{\prime}$ & 414 & {$[27]$} \\
\hline & Bacphy-1R & $5^{\prime}$ ggtaaggttcctcgcgta $3^{\prime}$ & & \\
\hline \multirow[t]{2}{*}{ Firmicutes } & Firmi-1F & $5^{\prime}$ ctgatg gag caacgccgcgt $3^{\prime}$ & 429 & {$[28]$} \\
\hline & Firmi-1R & $5^{\prime}$ acacytagy act cat cgttt $3^{\prime}$ & & \\
\hline \multirow[t]{2}{*}{ Proteobacteria } & Proteo-F & $5^{\prime}$ cacttcaacctgatcacctac c $3^{\prime}$ & 528 & {$[27]$} \\
\hline & Proteo-R & $5^{\prime}$ gcagcgggtccatgt tat $3^{\prime}$ & & \\
\hline \multirow[t]{2}{*}{ Actinobacteria } & Actino-F & $5^{\prime}$ gtyaactcg gag gaaggt $3^{\prime}$ & 195 & {$[22]$} \\
\hline & Actino-R & $5^{\prime}$ ctgatctgc gat tac tag cgactc $\mathrm{c} 3^{\prime}$ & & \\
\hline \multirow[t]{2}{*}{ Latobacillus } & Lacto-F & $5^{\prime}$ cttgtacacaccgeccgtca $3^{\prime}$ & 248 & {$[21]$} \\
\hline & Lacto-R & $5^{\prime}$ ctcaaa act aaacaaagt $3^{\prime}$ & & {$[23]$} \\
\hline \multirow[t]{2}{*}{ Bifidobacteria } & Bifi-1F & $5^{\prime}$ tcgcgtcyggtgtgaaag $3^{\prime}$ & 243 & {$[25]$} \\
\hline & Bifi-1R & $5^{\prime}$ cca cat ccagcrtcc ac $3^{\prime}$ & & \\
\hline \multirow[t]{2}{*}{ Faecalibacterium } & Fprau-1F & $5^{\prime}$ ggaggattgacccettca gt $3^{\prime}$ & 203 & {$[29]$} \\
\hline & Fprau-1R & $5^{\prime}$ ctggtcccgaagaaacac at $3^{\prime}$ & & \\
\hline \multirow[t]{2}{*}{ Roseburia } & Rosb-F & $5^{\prime}$ tactgcattggaaactgt cg $3^{\prime}$ & 230 & {$[30]$} \\
\hline & Rosb-R & $5^{\prime}$ cggcaccgaagagca at $3^{\prime}$ & & \\
\hline \multirow[t]{2}{*}{ Escherichia } & Esc-F & $5^{\prime}$ cat gccgcgtgtatgaagaa $3^{\prime}$ & 96 & {$[24]$} \\
\hline & Esc-R & $5^{\prime}$ cgggtaacgtcaatgagcaaa $3^{\prime}$ & & \\
\hline \multirow[t]{2}{*}{ Klebsiella } & Kleb-F & $5^{\prime}$ gga tat ctgaccagtcgg $3^{\prime}$ & 176 & {$[31]$} \\
\hline & Kleb-R & $5^{\prime}$ gggttttgcgtaatgatctg $3^{\prime}$ & & \\
\hline
\end{tabular}

PCR conditions were further optimized by gradient PCR using metagenomic DNA as a template to select the best optimum temperature/time conditions of annealing for quantitative real-time PCR. The PCR cycling conditions for $16 \mathrm{~S}$ rRNA universal primers for the different set of primers included initial denaturation of $95{ }^{\circ} \mathrm{C}$ for $5 \mathrm{~min}$ and 35 cycles each of denaturation $95^{\circ} \mathrm{C} / 30 \mathrm{~s}$, annealing $\left(52{ }^{\circ} \mathrm{C}, 54{ }^{\circ} \mathrm{C}, 56{ }^{\circ} \mathrm{C}, 58{ }^{\circ} \mathrm{C}, 60{ }^{\circ} \mathrm{C}\right.$ and $\left.62{ }^{\circ} \mathrm{C}\right) / 30 \mathrm{~s}$ and extension $72{ }^{\circ} \mathrm{C} / 30 \mathrm{~s}$ with final extension at $72{ }^{\circ} \mathrm{C}$ for $7 \mathrm{~min}$. The amplification was checked by visualizing the gel under Gel documentation System (G box syngene).

Further, the melt curve analysis for validation of primer specificity in real-time PCR was performed in Roche, Light Cycler under optimized conditions from the randomly selected samples as template. The qPCR cycling conditions comprised of denaturation $\left(95{ }^{\circ} \mathrm{C} / 10 \mathrm{~s}\right)$, annealing (at the respective optimized temperature/20 s) and extension $\left(72{ }^{\circ} \mathrm{C} / 20 \mathrm{~s}\right)$, repeated for 40 cycles and the melt curve analysis was done at a temperature range of 60-95 ${ }^{\circ} \mathrm{C}$. The real-time PCR products were further separated on gel electrophoresis (2\% agarose gel) and visualized under UV light after ethidium bromide staining in Gel-Doc system for conforming amplification.

\section{Absolute quantification of target bacterial groups}

Compared to relative quantification, the standard-curve (SC) based absolute quantification is frequently used in gut microbial studies for various kinds of analysis like gut microbial compositional analysis, their comparative abundance, and to compute Bacteroidetes to Firmicutes ratio. All the Molecular Biology techniques which include PCR amplification, purification of PCR products, ligation, transformation and plasmid minipreps, etc. were performed as per the protocols given by [32] as well as the manufacturer's instructions.

The conventional PCR was used to prepare target DNA for the quantification of total bacteria (Eubacteria), and the various target phyla and genera involved in the study. PCR amplicons of all the major gut enterotypes were prepared by setting up a $25 \mu \mathrm{l}$ PCR reaction using optimized PCR conditions and amplicons obtained were electrophoresed on agarose gels (1.8\%) for further confirmation of amplification prior to the purification. The amplified products of target DNA of gut enterotypes were purified in Wizard SV Gel and PCR Clean-up system using manufacture's protocol. The purified standard target DNA for each of the target bacterial phyla and genera was ligated into pGEM $^{\circledR}-\mathrm{T}$ Easy Vector, 
one of the widely used convenient system to clone PCR products generated by certain thermostable polymerases. The ligation was performed at refrigeration temperature for overnight before using it for calcium chloride induced transformation. The ligated product was transformed in calcium chloride induced competent $E$. coli $\mathrm{DH} 5 \alpha$ cells to obtain plasmid clones containing inserted standard target DNA [32].

The transformants obtained on LB agar plates with ampicillin were picked up and inoculated in LB broth containing ampicillin and incubated in shaking incubator at $37{ }^{\circ} \mathrm{C}$ for $12-16 \mathrm{~h}$ after which the cells were pelleted and used for plasmid isolation. Plasmid from respective transformed clones of DH5 $\alpha$ cells was extracted from randomly picked colonies using Gen Elute Plasmid Miniprep Kit (Sigma), separated by agarose gel electrophoresis $(0.8 \%)$ and visualized under Gel Doc system. Further, the cloned copies were also confirmed for the presence of standard target DNA by PCR using primers specific for the target genes. The concentration of cloned plasmids was determined and the copy number of target DNA was calculated. The cloning yielded plasmid copies in the range from $4.97 \times 10^{9}$ to $1.34 \times 10^{10}$, which were sufficient in number to construct standard curve in the downstream data analysis.

\section{Calculation of plasmid copy number}

Copy number of the standard target gene was calculated using the formula

Number of copies $=\left(\right.$ amount $\left.\times 6.022 \times 10^{23}\right) /$

$$
\text { (length } \left.\times 1 \times 10^{9} \times 650\right) \text {. }
$$

For preparation of standard curve, the plasmid copies containing standard DNA of target gut enterotype was tenfold serially diluted in the range of $1 \times 10^{4}-1 \times 10^{9}$ copies/ $\mu \mathrm{l}$, to be used as template in the quantitative real-time PCR.

\section{Construction of standard curve for absolute quantification}

For preparation of standard curve, the plasmid copies containing standard DNA of target gut enterotypes (tenfold serially diluted in the range of $1 \times 10^{4}-1 \times 10^{9}$ copies $\left./ \mu \mathrm{l}\right)$ and the test metagenomic DNA from all the healthy, MAM and SAM children were used as template in the quantitative realtime PCR. The reactions were carried out in a 96 well Light Cycler and was set up in the following manner. One vial each of SYBR Premix Ex Taq II (TaKara, Japan), water (PCR grade) and working PCR primers (10 $\mu \mathrm{M}$ concentration) were thawed over ice. The qPCR $(10 \mu \mathrm{l})$ reaction comprised of PCR grade water $(3.4 \mu \mathrm{l})$; SYBR Green $(2 \mathrm{x}, 5.0 \mu \mathrm{l})$; Primer F and R ( $10 \mathrm{pmol} / \mu \mathrm{l}, 0.3 \mu \mathrm{l}$ each) and template DNA $(1.0 \mu \mathrm{l})$. The qPCR programme included the following steps pre-incubation $\left(95^{\circ} \mathrm{C} / 300 \mathrm{sec}\right)$, Denaturation $\left(95^{\circ} \mathrm{C} / 30 \mathrm{sec}\right)$, Annealing $\left(56{ }^{\circ} \mathrm{C} / 30 \mathrm{sec}\right)$, Extension $\left(72^{\circ} \mathrm{C} / 30 \mathrm{sec}\right)$, Melting $\left(95^{\circ} \mathrm{C} / 10 \mathrm{sec}, 65^{\circ} \mathrm{C} / 60 \mathrm{sec}, 97^{\circ} \mathrm{C} / 1 \mathrm{sec}\right)$ and Cooling $\left(37^{\circ} \mathrm{C} / 30 \mathrm{sec}\right)$ the above steps were repeated for 45 cycles. The $\mathrm{C}_{\mathrm{q}}$ values for each dilution of standard target DNA was measured in duplicate in real-time quantitative PCR, using respective dilution of plasmid copies as template for specific set of primers for target gut microbial enterotypes. The obtained $\mathrm{Cq}$ values were plotted against the logarithm of their template copy numbers of each standard DNA and the standard curve was generated by a linear regression of the plotted points.

\section{Metagenome analysis of faecal samples through high-throughput amplicon sequencing}

Faecal metagenomic DNA from two samples i.e., one each from healthy and SAM groups were sent for amplicon sequencing. 16S rRNA gene (V3-V4 region) sequencing of faecal metagenomic DNA samples was performed with MiSeq Illumina platform to identify the Operational Taxonomic Units (OTU's) and to compare the gut microbial abundance among the various groups of children i.e., healthy and SAM considered in the study.

The Illumina paired end reads $(270 \times 2)$ were demultiplexed using bcl2fastq1 tool. The paired end reads were quality checked using FastQC2. The raw reads having primer sequence and high quality bases were selected. The reads were further stitched using Fastq-join3. These stitched reads were considered for further analysis using QIIME4 pipeline. The query sequences were clustered using UCLUST5 method. The taxonomy of these clusters was assigned based on $>=97 \%$ sequence similarity against the curated chimera free 16S rRNA database (Greengenes $6 \mathrm{v}$ 13.8).

\section{Statistical analysis}

\section{Statistical analysis for sample size determination.}

The sample size was determined by running a Power analysis with the $G^{*}$ Power 3.1.9.7 software considering the following parameters, Effect size $f=0.25$ (medium); Type I error probability $(\alpha=0.05)$; Power $(1-\beta$ err prob $)=0.80$; Number of groups $=3$. The power analysis with the $F$ test and One way ANOVA test statistic, yielded the total sample size of 159 . However, this study being a proof of concept (pilot study), we restricted our sample size to 30 i.e., 10 samples in each group of healthy, MAM and SAM. 


\section{Normality tests}

The Normality tests were performed to know if the selected samples where from a population following Gaussian distribution. The GraphPad PRISM version 9 was used to perform the normality tests. Both the D'Agostino and Pearson omnibus normality test and Shapiro-Wilk normality test were performed with a $p$ value of 0.05 . The samples passed both the normality tests (D'Agostino and Pearson omnibus normality test, $p$ value: $0.385,0.670,0.282$ for healthy, MAM and SAM respectively. Similarly, Shapiro-Wilk normality test, $p$ value: $0.584,0.586,0.062$ for healthy, MAM and SAM respectively).

\section{Statistical analysis of absolute quantification data of gut bacteria}

\section{Univariate analysis}

The standard curve for absolute quantification was generated using the Microsoft Office Excel package. The log copy number of cells in the samples were determined by solving the straight-line equation of the standard curve. The data pertaining to the log copy number of cells for each target phyla/genera comprising the three categories of subjects i.e., healthy, MAM and SAM were compared using GraphPad PRISM version 9. One-way ANOVA was applied as a test statistic to compare the means among the three group of subjects at 5\% level of significance. The Tukey's Multiple Comparison test was used as a post hoc test in cases where significant results were observed, to identify the pairs of groups that gave significant differences.

\section{Multivariate analysis}

The multivariate analysis like unsupervised Principal Component Analysis (PCA) and supervised Partial Least Square Discriminant Analysis (PLS-DA) were performed with the MetaboAnalyst 4.0.

\section{Results}

\section{Selection of children for the study}

The $\mathrm{Z}$ score is a widely used parameter to differentiate the healthy from the malnourished children. Based on $\mathrm{Z}$ score, samples from a total of 30 children i.e., 10 in each category, healthy (10), MAM (10) and SAM (10), were considered for the study. The average age of children in each group was $3.01 \pm 1.11$ (healthy), $3.66 \pm 0.89(\mathrm{MAM})$ and $2.99 \pm 0.68$ (SAM) years. Similarly, the average $Z$ score was recorded to be $-0.21 \pm 0.6,-2.25 \pm 0.79$ and $-3.58 \pm 0.55$ in healthy, MAM and SAM groups of children respectively (Table S1).

\section{Extraction of metagenomic DNA from faecal samples}

The metagenomic DNA extraction using Zymo Research Mini Prep. Kit yielded DNA of sufficient quantity and satisfactory purity for downstream applications like PCR, cloning and subsequent absolute quantification of targeted bacterial groups using Real Time PCR (qPCR). The average concentrations of the extracted metagenomic DNA for healthy, MAM and SAM children were found to be $111.23 \pm 42.84,129.56 \pm 58.95$ and $181.69 \pm 62.19 \mathrm{ng} /$ $\mu \mathrm{l}$ respectively. The purity of the DNA was in the range of 1.75-1.85. Running a $0.8 \%$ agarose gel for analyzing integrity of DNA yielded satisfactory results as depicted in Fig. S2.

Further, the PCR amenability of metagenomic DNA determined through $16 \mathrm{~S}$ rRNA gene amplification from all the selected 30 samples, yielded PCR amplicons of size $\sim 1.5 \mathrm{~kb}$ as illustrated in Fig. S3., which confirms the PCR amenability of metagenomic samples to be used in absolute quantification of bacterial groups using qPCR.

\section{Validation of primers for target gut bacteria}

Real-time qPCR melt curve analysis conducted with all the selected primers, to ascertain their specificity under optimized conditions using metagenomic DNA, yielded single peak at a particular melting temperature. The clear and specific bands obtained by running the real-time PCR products on a $2 \%$ agarose gel further confirmed the specificity. The PCR amplification using the target phyla/genera specific primers of (Bacteroidetes, Firmicutes, Proteobacteria, Actinobacteria, Lactobacilli, Bifidobacteria, Faecalibacteria, Roseburia, Escherichia and Klebsiella) delivered expected results by yielding products of expected amplicon sizes (Fig. S4).

\section{Absolute quantification of target bacterial groups}

For absolute quantification of target bacterial groups, PCR products of specific bacterial groups were purified, ligated into pGEMT-easy vector and subsequently transformed into $E$. coli DH5 $\alpha$. The recombinant plasmids were extracted from respective clones of DH5 $\alpha$ cells on visualization under Gel Doc (Fig. S5). The screening for target DNA, among the extracted plasmids by PCR using primers specific for the target genes, yielded amplicons of respective product sizes as illustrated in (Fig. 
S6), which further confirmed the successful transformation. The concentration of cloned plasmids that was used to determine the copy number of target DNA showed that the cloning yielded plasmid copies in the range of $4.97 \times 10^{9}-1.34 \times 10^{10}$, sufficient in number to construct standard curve in the downstream data analysis.

The standard curve was derived as per the procedure mentioned earlier in the Methods Section (Construction of standard curve for absolute quantification). From the slope of each standard curve, PCR amplification efficiency $(E)$ was calculated according to the equation (Rasmussen 2001): $E=10^{-1 / \text { slope }}$. All the standard curves constructed for the target gut microbial groups showed the $R^{2}$ Values in the range of 0.9819-0.9999 with the respective slope values within the accepted range from -3.32 to -2.5 . The above trend hints at a superior efficiency (nearly equal to 1 or $100 \%$ efficiency) in the amplification of standard target DNA with varied concentrations (Table S2).

The results of absolute log copy numbers of all the standard target DNA of the selected gut bacteria [total bacteria (Eubacteria), Bacteroidetes, Firmicutes, Proteobacteria, Actinobacteria, Lactobacilli, Bifidobacteria, Roseburia, Faecalibacteria, Escherichia and Klebsiella] were derived from the average of obtained $\mathrm{C}_{\mathrm{q}}$ values (in duplicate), using the linear regression equation deduced out of the standard curve. The results have been tabulated below (Table 2) and have also been graphically elucidated as univariate data in Fig. 1 for targeted bacterial phyla and Fig. 2 for targeted bacterial genera.

\section{Comparison of abundance through Univariate Analysis}

In comparative gut microbiota studies, it is essential to enumerate the total gut bacterial abundance and diversity in the study populations. Hence in our study, we compared Eubacterial abundance in healthy, MAM and SAM children. However, on comparison, no significant differences in the total abundance of Eubacteria was reported among the three nutritional groups as is evident from Fig. 1a. Similarly, no significant differences were observed in the abundance of major bacterial phyla like Bacteriodetes (Fig. 1b) and Firmicutes (Fig. 1c). Nevertheless, Actinobacteria were significantly the most abundant in the Healthy gut in comparison to the malnourished (SAM) gut as depicted in Fig. 1e, whereas in case of phylum Proteobacteria, no significant difference was observed in relation to its abundance between healthy and SAM gut (Fig. 1d).
Table 2 Absolute log copy numbers of all the standard target DNA of the selected gut bacteria

\begin{tabular}{|c|c|c|c|c|c|}
\hline Phyla/Genera & Healthy & MAM & SAM & Statistical significance & Figure \\
\hline \multicolumn{6}{|l|}{ Phyla } \\
\hline Eubacteria & $8.58 \pm 0.17$ & $8.71 \pm 0.11$ & $8.68 \pm 0.12$ & $\begin{array}{l}\text { Non-significant } \\
(P>0.05)\end{array}$ & 1(a) \\
\hline Bacteroidetes & $7.75 \pm 0.71$ & $8.10 \pm 0.59$ & $8.35 \pm 0.31$ & $\begin{array}{l}\text { Non-significant } \\
(P>0.05)\end{array}$ & 1(b) \\
\hline Firmicutes & $6.83 \pm 0.60$ & $7.24 \pm 0.47$ & $6.79 \pm 0.28$ & $\begin{array}{l}\text { Non-significant } \\
(P>0.05)\end{array}$ & $1(\mathrm{c})$ \\
\hline Proteobacteria & $5.81 \pm 1.24$ & $5.88 \pm 0.64$ & $5.35 \pm 1.20$ & $\begin{array}{l}\text { Non-significant } \\
(P>0.05)\end{array}$ & $1(d)$ \\
\hline Actinobacteria & $7.65 \pm 0.36$ & $7.41 \pm 0.38$ & $7.13 \pm 0.47$ & $\begin{array}{l}\text { Significant decrease } \\
(P<0.05)\end{array}$ & $1(\mathrm{e})$ \\
\hline \multicolumn{6}{|l|}{ Genera } \\
\hline \multicolumn{6}{|l|}{ Probionts } \\
\hline Lactobacillus & $3.70 \pm 1.89$ & $6.32 \pm 1.28$ & $5.95 \pm 0.93$ & $\begin{array}{l}\text { Significant increase } \\
(P<0.05)\end{array}$ & 2(a) \\
\hline Bifidobacteria & $7.50 \pm 0.41$ & $7.15 \pm 0.41$ & $6.73 \pm 0.97$ & $\begin{array}{l}\text { Significant decrease } \\
(P<0.05)\end{array}$ & 2(b) \\
\hline \multicolumn{6}{|l|}{ Butyrogenic } \\
\hline Faecalibacterium & $4.55 \pm 0.97$ & $5.04 \pm 0.53$ & $5.15 \pm 0.38$ & $\begin{array}{l}\text { Non-significant } \\
(P>0.05)\end{array}$ & $2(\mathrm{c})$ \\
\hline Roseburia & $5.83 \pm 1.64$ & $6.73 \pm 0.97$ & $6.59 \pm 0.85$ & $\begin{array}{l}\text { Non-significant } \\
(P>0.05)\end{array}$ & $2(d)$ \\
\hline \multicolumn{6}{|l|}{ Pathogenic } \\
\hline Escherichia & $6.73 \pm 0.96$ & $6.40 \pm 0.68$ & $5.28 \pm 0.00$ & $\begin{array}{l}\text { Significant decrease } \\
(P<0.05)\end{array}$ & $2(\mathrm{e})$ \\
\hline Klebsiella & $3.86 \pm 1.16$ & $3.45 \pm 1.23$ & $2.88 \pm 0.89$ & $\begin{array}{l}\text { Non-significant } \\
(P>0.05)\end{array}$ & $2(\mathrm{f})$ \\
\hline
\end{tabular}




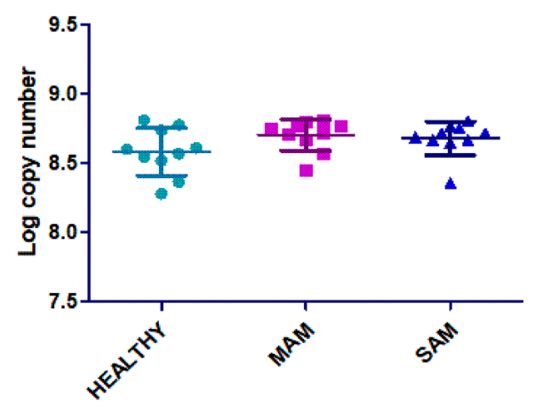

a Eubacteria $[P>0.05]$

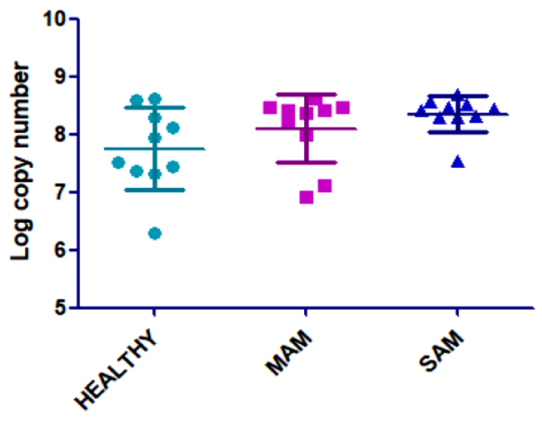

b Bacteroidetes $[\mathrm{P}>0.05]$

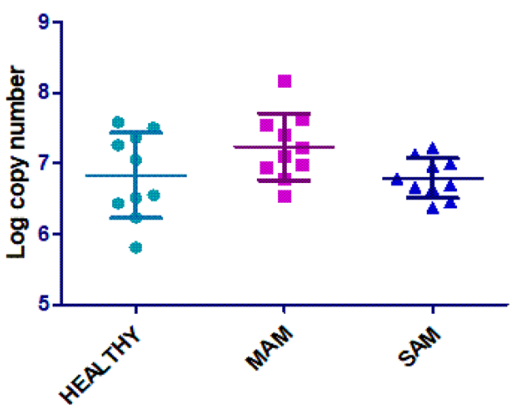

C Firmicutes $[P>0.05]$
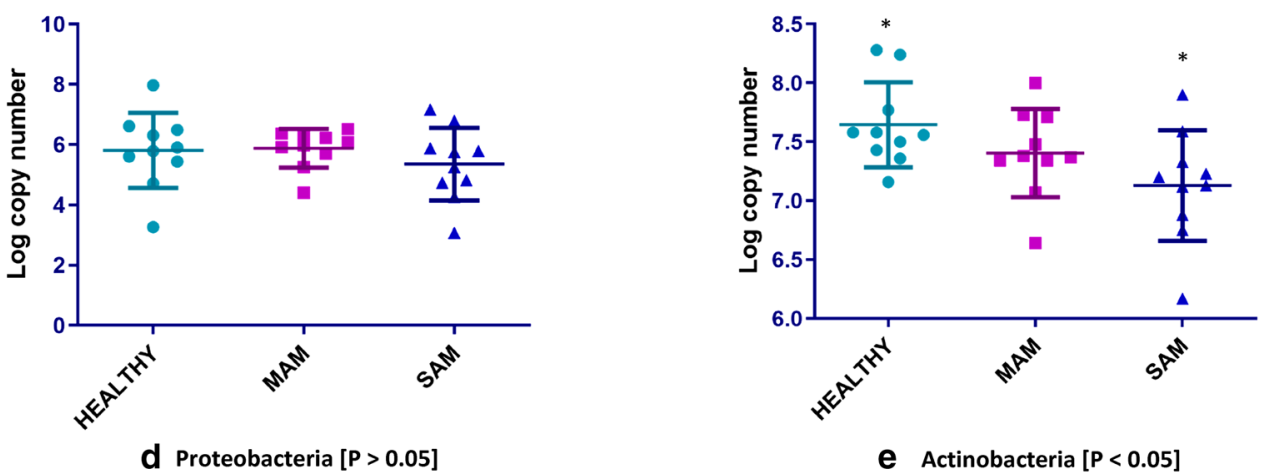

Fig. 1 Comparison of bacterial abundance (Phyla level) among Healthy, MAM and SAM subjects. *Indicates significance (@0.05) among the groups

At the genus level, interesting trends were observed. A significant difference was encountered in the abundance of Bifidobacteria, wherein the healthy child gut had a higher abundance of these probionts in comparison to their malnourished (SAM) counterparts (Fig. 2b). However, an inverse trend was observed with other important probiotic genera Lactobacillus with its significant higher abundance in the malnourished gut (SAM and MAM) in comparison to the healthy gut (Fig. 2a). Significant differences were also encountered in the abundance of Escherichia genera, wherein the healthy gut was found to have significantly higher proportion of Escherichia in comparison to the gut of SAM children as depicted in Fig. 2e. Though differences in abundance were observed in other genera of Faecalibacterium, Roseburia and Klebsiella, these differences were not significant between different nutritional groups (Fig. 2c, d and $\mathrm{f}$.

\section{Comparison of abundance through Multivariate Analysis}

Apart from the univariate analysis, multivariate analysis methods like PCA and PLS-DA were subsequently employed to understand the gut microbiota patterns or clusters associated study populations (healthy, MAM and SAM children) based on the observations (log copy number of targeted bacterial genera) recorded with the individual samples under the study category. The individual samples were represented by isolated points in the PCA score plot, and the study populations were clustered into three separate regions depending on their similarities. On analysis, the three nutritional groups i.e., Healthy, MAM and SAM were not clearly distinct at the Phylum level, however the discrimination was more pronounced at the Genera level as depicted in Figs. 3a and 4 a respectively. These trends suggest certain changes in the abundance of bacterial genera among the Healthy, MAM and SAM population. Besides, PCA Biplots revealed that a greater proportion of healthy samples are aligned towards Actinobacteria phyla and the same trend was also reflected for the Bifidobacteria genera of the same phylum group as elucidated in Figs. 3b and 4b, respectively.

Later, partial least squares discriminant analysis (PLSDA), a supervised pattern recognition model was used to reveal microbiota variation between different nutritional groups (healthy, MAM and SAM) in the score plots (Fig. 5a, b). Each dot in this score plot depicts an observation of individual sample, and the distance between two dots reflects the similarity of the sample composition. The score plot of PLS-DA at the Phyla level (Fig. 5a) clustered the samples to a greater extent, hence is more discriminative than the one revealed by PCA. The more 


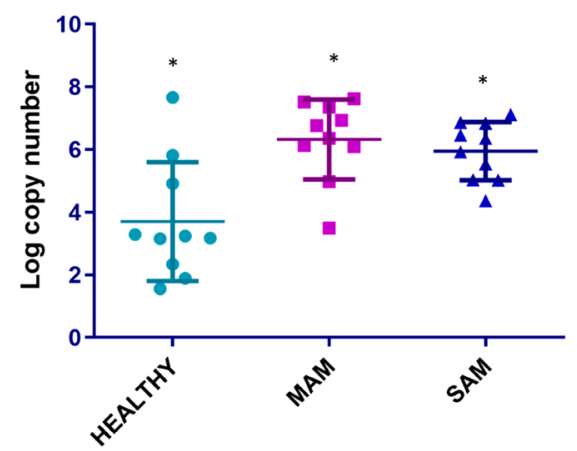

a Lactobacillus $[* \mathrm{P}<0.05]$

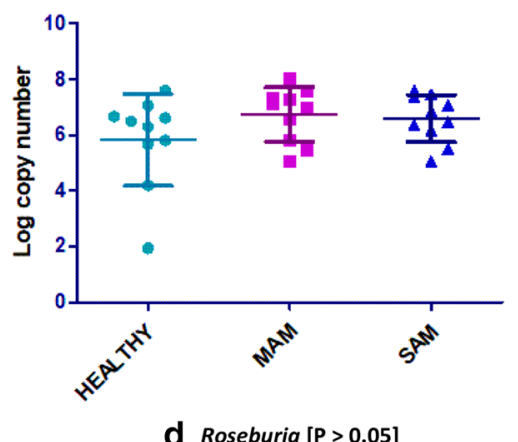

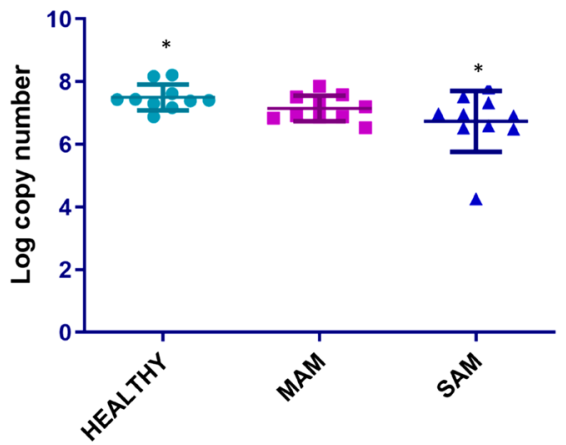

b Bifidobacteria [ $\left.{ }^{*} \mathrm{P}<0.05\right]$

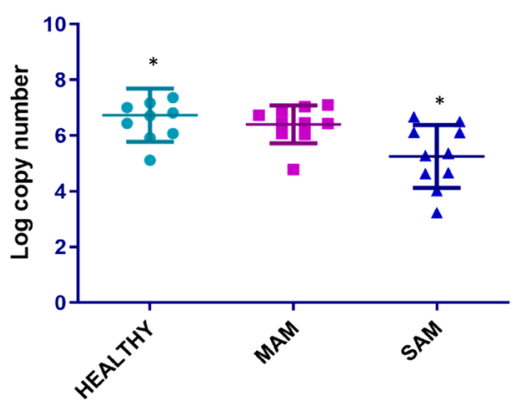

e Escherichia $\left[{ }^{*} \mathrm{P}<0.05\right]$

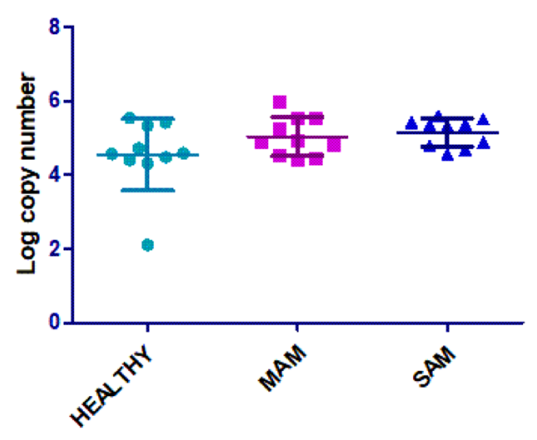

C Faecalibacterium $[\mathrm{P}>0.05]$

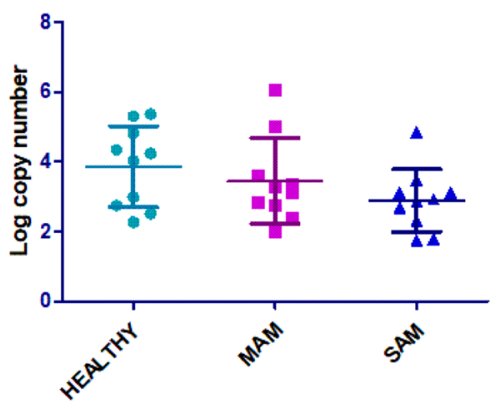

f Klebsiella $[\mathrm{P}>0.05]$

Fig. 2 Comparison of bacterial abundance (Genera level) among Healthy, MAM and SAM subjects. *Indicates significance (@0.05) among the groups

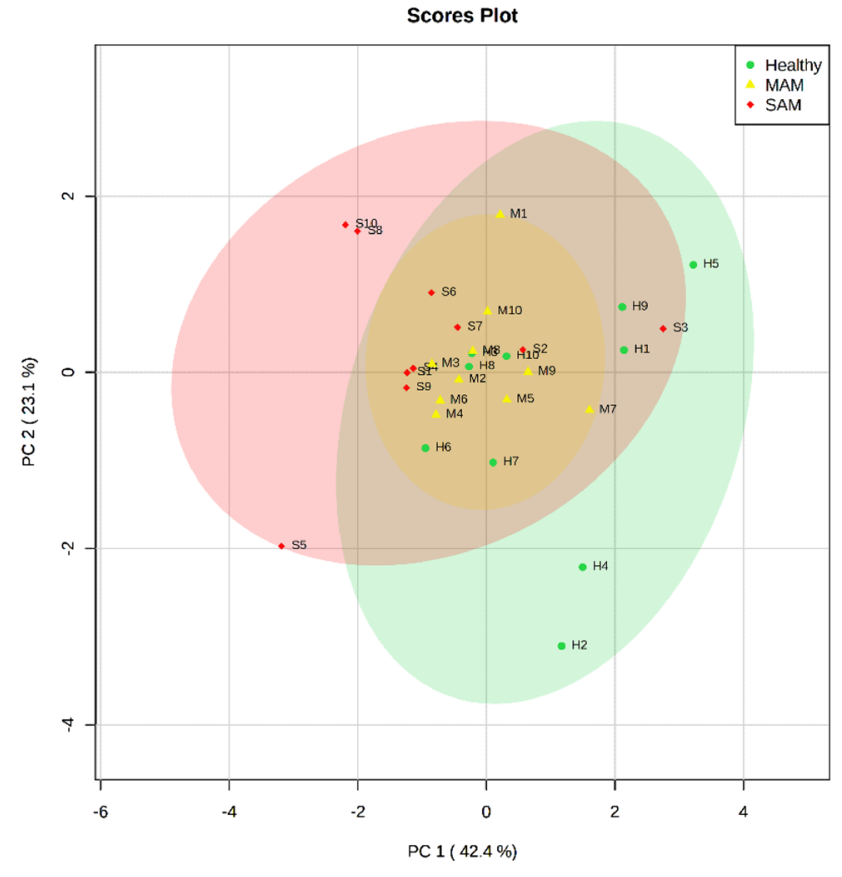

a PCA 2D Score Plot

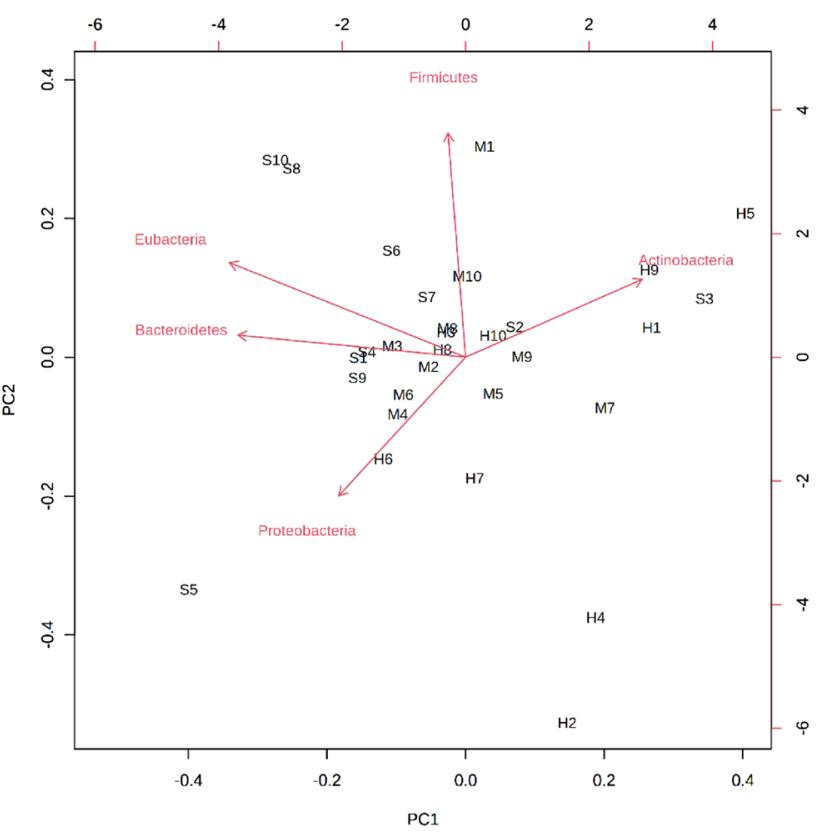

b PCA Biplot

Fig. 3 a PCA 2D Score Plot (Phyla Level). b PCA Biplot (Phyla level) 


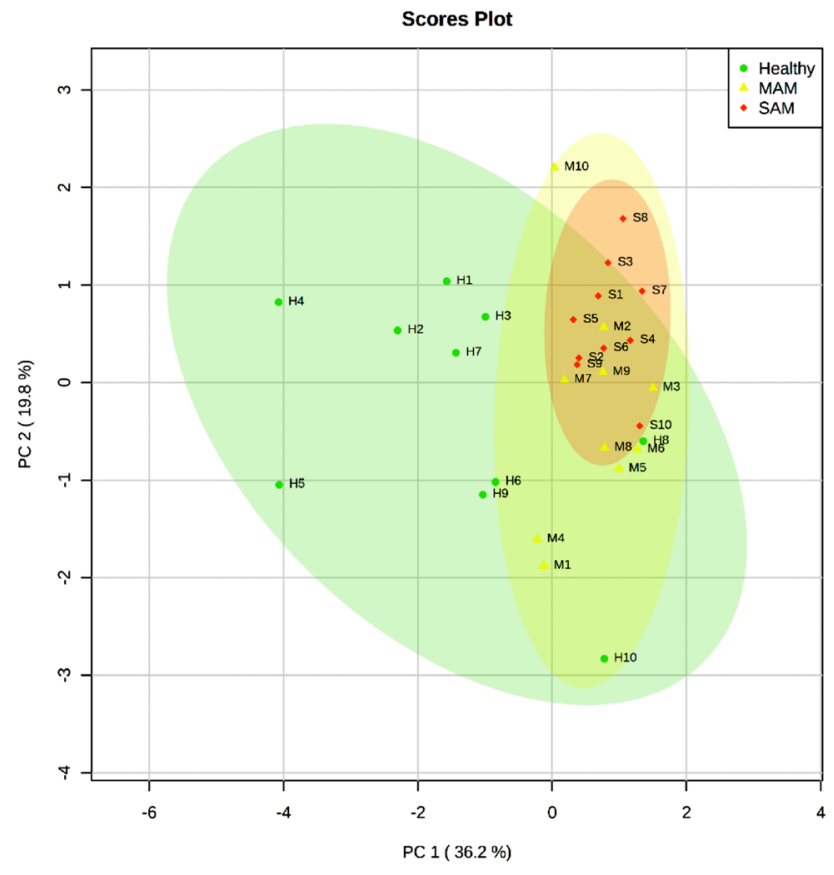

a PCA 2D Score Plot

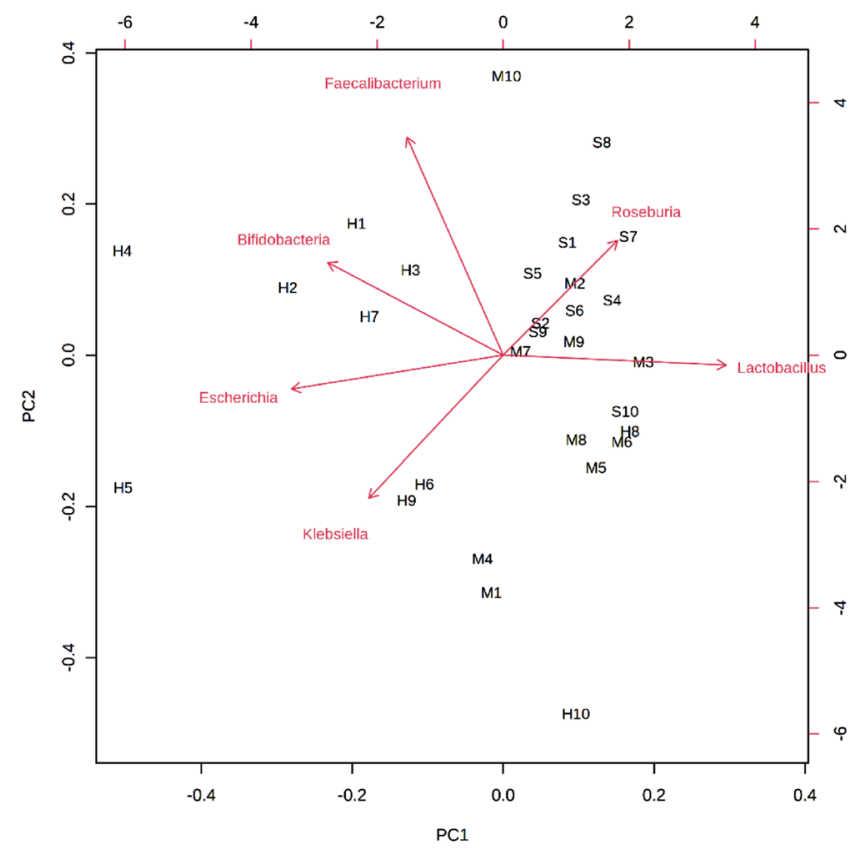

b PCA Biplot

Fig. 4 a PCA 2D Score Plot (Genera Level). b PCA Biplot (Genera Level). PCA was performed on log-copy number of absolute qPCR data on bacterial genera to assess the bacterial composition similarity between healthy, MAM and SAM children

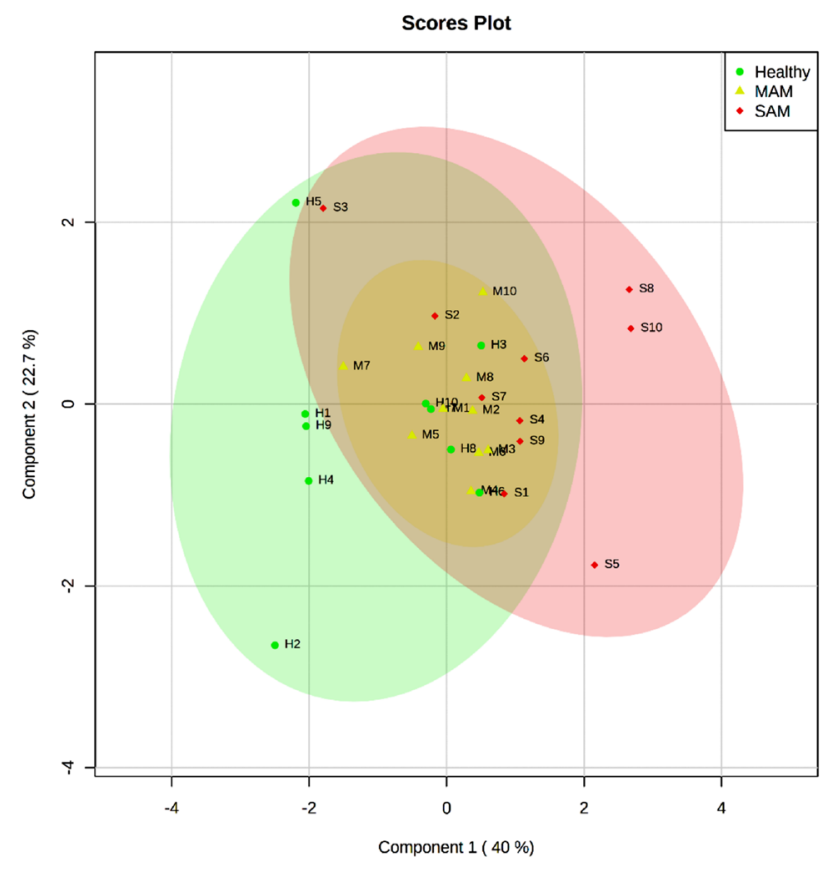

a PLS-DA 2D Score Plot [Phyla Level] *

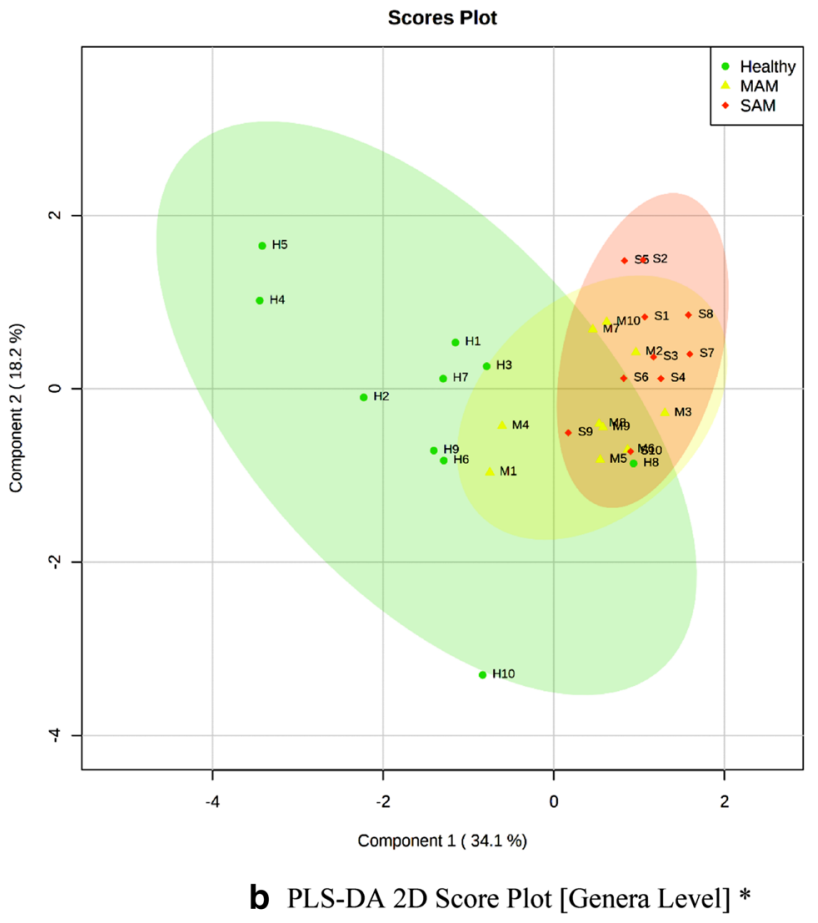

Fig. 5 a PLS-DA 2D Score Plot (Phyla level)*. b PLS-DA 2D Score Plot (Genera level)*. *PLS-DA was performed on log-copy number of absolute qPCR data on bacterial phyla and genera to assess the bacterial composition similarity between healthy, MAM and SAM children 
pronounced separation of nutritional groups at genera level as visualized by the 2D score plots of PLS-DA further indicated variation in the abundance of gut bacterial genera between the nutritional groups (Fig. 5b). Hence, it can be concluded that the gut bacterial genera showed varied abundance in children of different nutritional health status.

\section{Faecal metagenome 16S rRNA illumina sequencing}

The 16S rRNA amplicon sequencing and its resultant data analysis was done only for two samples that is one each of healthy and SAM. Such an approach was selected based on our absolute quantification data, as no significant changes could be found between healthy vs MAM as well as MAM vs SAM. Hence, we performed the sequencing only for the extreme cases i.e., healthy and SAM and the data pertaining to that has been illustrated in Fig. 6a and b respectively. The bacterial taxonomy results obtained from QIIME (Quantitative Insights Into Microbiological Ecology), the widely used software tool in microbial diversity and taxonomy analysis were also compared for the abundance of bacterial groups at higher and lower taxonomic levels like phylum and genera as shown in Fig. 6a and b.

\section{Bacterial abundance at the Phyla level}

The bacterial abundance analysis at the phylum level revealed Firmicutes, Bacteroidetes, Actinobacteria and Proteobacteria as major gut phyla; however, their abundance was quite different between healthy and SAM (Fig. 6a). Among these phyla, Firmicutes was identified as major phylum in both healthy and SAM nutritional group of children with respective contributions of $55.77 \%$ and $57.09 \%$ to total bacterial phyla. Bacteroidetes was recorded as second major phylum with percentage contribution of $34.83 \%$ and $32.97 \%$ to the total phyla of healthy and SAM children, respectively. However, the abundance of phylum Actinobacteria was considerably distinct between healthy and SAM children as its predominance was fairly higher in healthy $(8.83 \%)$ children compared to SAM (1.69\%) children. Conversely, the abundance of Proteobacteria was rationally higher in SAM (7.53\%) as compared to healthy children, where its abundance is less than one percent $(0.34 \%)$.

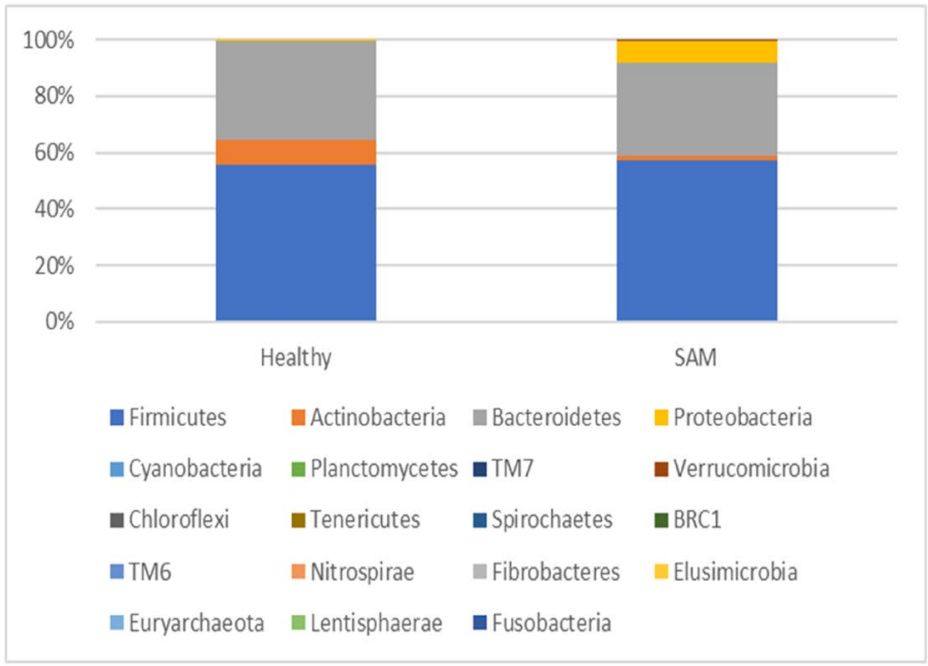

a Comparative abundance at the Phyla Level

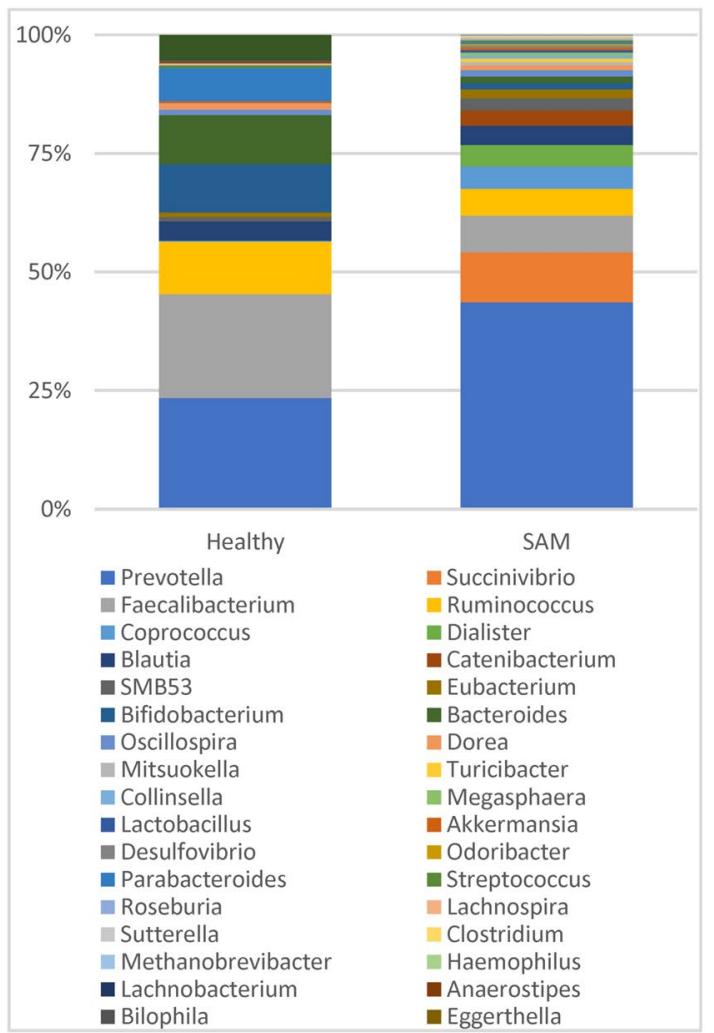

b Comparative abundance at the Genera Level

Fig. 6 a Comparative abundance at the Phyla level. b Comparative abundance at the Genera Level 


\section{Bacterial abundance at the Genera level}

In our analysis pertaining to gut bacterial abundance at the genera level, we encountered a total of approximately 55 genera in the healthy and 80 genera in SAM sample. Among these, the dominant genera (having contribution $>1 \%$ ) in healthy gut were of Prevotella (23.42\%), Faecalibacterium (21.88\%), Ruminococcus (11.05\%), Bifidobacterium (10.12\%), Bacteroides (10.40\%), Blautia (4.05\%), Oscillospira (1.07\%) and Dorea (1.44\%). Similarly, in SAM gut, Prevotella (43.63\%), Faecalibacterium (7.77\%), Ruminococcus (5.63\%), Bifidobacterium (1.36\%), Bacteroides (1.35\%), Blautia (4.05\%), Oscillospira (1.32\%) and Dorea (0.96\%) were recorded as major genera. On comparison of abundance data of different bacterial genera, butyrogenic bacteria such as Bifidobacteria and Faecalibacterium were found to be considerably higher in the healthy gut in comparison to the SAM gut. Besides, other commensal genera like $\mathrm{Bac}$ teroides and Ruminococcus showed the similar trend with higher abundance in healthy gut. However, the other bacterial genera of health importance, Lactobacillus depicted an anomalous trend, with its higher abundance $(0.55 \%)$ in the SAM gut in comparison to the healthy gut $(0.10 \%)$ as elucidated in Fig. 6 b.

\section{Discussion}

The human gut harbors a plethora of diverse microbes, forming a complex ecological niche that is influenced by several host and environmental factors. Of all the exogenous factors affecting gut microbiome, a long-term diet and nutrition appears to have the largest effect to date [33, 34]. Often, decreased gut microbial diversity has been found to be associated with the poor nutritional status of the host (children) [4]. Further, it has also been observed that children suffering from SAM during their early years of life experience significant delays in shaping of a healthy gut microbiota [35]. The subtle changes in microbiota composition at the early developmental stages of life lead to "dysbiosis" characterized by the enhancement of gut permeability which ultimately leads to inflammation [36, 37]. Alternatively, chronic inflammation triggered by increased susceptibility to recurrent enteric pathogens could also lead to villous atrophy, anorexia and finally cause impaired growth ending up in malnutrition [19, 38].

To assess the actual difference in microbial diversity, it was imperative to enumerate the total bacterial content. On comparing the results of total abundance of Eubacteria, with previous observations, we found subtle differences in linking decreased Eubacterial population with the poor nutritional status of children as reported by [2] through metagenomic approach and [18] by culturomics approach. All of this difference could be due to the differences in the region and size of the population selected for the study and use of different quantification approaches i.e., "High Throughput Sequencing" (HTS), q-PCR and "Culturomics" used in these studies.

Bacteriodetes and Firmicutes are the two predominant human gut phyla [39] and non-significant increase in abundance of both Bacteriodetes and Firmicutes was observed in MAM and SAM children. This result was consistent with the previous study of gut microbiota of healthy and malnourished (Kwashiorkor) children by 'culturomics' approach [18]. However, in other studies [3, 6] non-significant lower abundance of Bacteriodetes, higher predominance of Firmicutes was reported with increase in Firmicutes/Bacteroidetes ratio in healthy children.

The dominance of Proteobacteria compounded with decreased bacterial diversity was found to be detrimental to the postnatal maturation of gut microbiota [4, 7, 40, 41]. However, in our study, we observed its lower predominance in the malnourished [SAM] gut at non-significant level in similar lines with the results of other studies by [2] and Méndez-Salazar [6]. Contrary to this, the higher abundance (7.8\% and 17\%) of Proteobacteria in malnourished children was also reported in different studies carried out by [3, 9].

Members of Actinobacteria phylum are the early colonizers of infant gut [42]. A significant $(P<0.05)$ lower abundance of this phylum was observed in severely malnourished (SAM) gut in comparison to the Healthy gut. Our results are in agreement with those of [2] in malnourished children of India, [6] in Mexican children and [18] in African children who also observed depletion of Actinobacteria.

Being a vital component of intestinal microbiome, Lactobacillus spp. plays a pivotal role in the establishment of gut homeostasis besides improving the nutritional and immune health [43]. However, we observed a significantly higher abundance of Lactobacillus in the malnourished gut (MAM and SAM) in contradiction to the expected significant depletion of Lactobacillus in SAM children [2, 3, 5]. However, Alou et al. [18] reported higher dominance of Lactobacillus in malnourished children in culturomics study that is in similar lines to the results of our study though it was non-significant. These inconsistent results could be linked to the differences in diet and geography as discussed earlier in this study.

Being a putative gut probiont, depletion of Bifidobacteria can pave the way for colonization of pathogenic genera [43]. Accordingly, the malnourished gut was found to be depleted in Bifidobacteria as reported by several other investigators i.e., $[2,3,5,7,40]$. These results clearly indicated that the proportion of Bifidobacteria, a signature bacterium of Healthy Mature Anaerobic Gut Microflora (HMAGM) varies between the healthy and malnourished gut. Besides, our data also implies that severe acute malnutrition leads 
to significant changes in the proportion of Bifidobacteria in the gut.

The genera Faecalibacterium and Roseburia are butyrogenic gut bacteria that are able to produce substantial amounts of butyrate in the gut. The quantification of abundance of these butyrogens becomes important and is often studied in research involving gut dysbiosis. However, no conclusions could be drawn as the results obtained were not significant. On comparison, our results were contradictory to those obtained by [40] who reported significant depletion of Roseburia and Faecalibacterium in the malnourished gut.

Escherichia belongs to commensal gut flora with some of its strains also known widely for their pathogenicity [44]. As the bacterium frequently colonizes human infants, it bears life long relationship with the host. Further, Klebsiella, a Gram-negative enteric pathogenic genus has also been found to be the most common cause of pneumonia among malnourished children [45]. The results obtained were contradictory to the expected higher abundance of Escherichia in MAM and SAM children [3, 40]. However, the declined abundance of Klebsiella in malnourished children [3] was also observed in our study at non-significant level.

The Multivariate analysis of PCA and PLS-DA revealed that the Healthy samples are different from the SAM samples on the basis of collective variables at Phyla and Genera level. The results of multivariate analysis supplement the findings of the univariate analysis where the significant differences in Actinobacteria and Bifidobacteria were observed between the Healthy and SAM samples as well as it also showed the significantly higher abundance of Lactobacillus in SAM samples. The same trend is reflected in the Multivariate analysis, especially at the genera level, the discrimination is more distinct in PLS-DA. In the Healthy samples, the variable Bifidobacteria is the major determining factor for the clustering of healthy samples. Similarly, Lactobacillus contributes majorly to the clustering of SAM samples.

Our study through the qPCR-based absolute quantification approach was also validated through $16 \mathrm{~S}$ rRNA amplicon sequencing approach with one healthy and one SAM sample. The results obtained from the 16S rRNA amplicon sequencing largely reiterate the findings of the qPCR based absolute quantification. The lowered abundance of Actinobacteria and Bifidobacteria in the SAM gut encountered by us was also reported by [5] in stunted Indian children and also by [15]. All the studies being referred to i.e. [2-6, 15] sampled children from low income families and resource poor neighborhoods of cities like Dhaka (Bangladesh) [3, 4]; Vellore, Kolkata (India) [2, 5]; Chimalhuacán (Mexico) [6] and Western African nations [7, 15]. In our study also, the sampled subjects from anganwadis, were from low income families and resource poor suburbs of Chandigarh. The poor sanitation and hygiene prevalent in such resource poor settings that cause environmental enteric dysfunction (EED), could also be a cause for the observed shifts in microbiome leading to the lowered abundance of anaerobes and a predominance of pathogens as observed in $[2-7,15]$. The diet could also bear a causal link to such a trend. The poor purchasing power of these low income families could lead to a diet poor in antioxidants probably due to the lack of access to antioxidant rich foods like fruits and vegetables, which could be a possible reason for a gut depleted in beneficial anaerobes as hypothesized by [15].

The lowered abundance of Actinobacteria and Bifidobacteria in the SAM gut encountered by us was also reported by [5] in stunted Indian children and also by [15]. However, results for Lactobacillus were contradictory to those reported by [4-6]. Lactobacillus genera though harboring the maximum number of probiotic bacteria is found to give the most discrepant results among several association studies. In most of the association studies between gut microbiota and other diseases, this genera presented a complex case of apparently inconsistent results [46]. Hence, it is possible that the same trend was replicated in association studies involving gut microbiota and malnutrition, as many studies [4-6] including ours gave contradictory results. One of the reasons for the observed lack of consistency could be the species specific or strain-specific effects of members of this genus.

The Proteobacteria phyla which showed an increased abundance in the SAM gut in several studies [2, 4-7, 40] was also found to reciprocate a similar trend in our study though these changes could only be perceived through the sequencing approach.

The differences in results obtained in various studies may be due to variation in sample size, region specific factors associated with gut microbiota, different dietary [food habits] and life style factors associated with the developmental programming of gut microbiota, host factors associated with their diversification, the different methodologies adopted for the metagenomic study and the large intra group variation in data points. However, the difference in the results of these studies is most likely due to methodological differences and differences in host cohorts (age, health status) and other dietary factors.

The current study though limited by a small sample size revealed interesting patterns wherein the gut bacterial profile varied with the nutritional status i.e. (healthy, MAM and SAM). Although, certain general presumptions can be made from the results of such gut microbiota study, the underlying biological mechanisms leading to such dysbiosis needs to be experimentally verified. Considering the small sample size, it would be premature to state that the findings support the hypothesis of a dysbiotic gut depleted mainly in Bifidobacteria, a signature bacterium responsible for shaping the Healthy Mature Anaerobic Gut Microbiota (HMAGM), as a probable contributing factor to childhood malnutrition. 
However, the insights gained from the current study can form the basis for more in-depth analysis of gut microbiomes involving larger populations of diverse nutritional status and geographies. Such studies would help to understand the role of specific microbes responsible for nutrient assimilation and their interactions that have an effect on host health. Further, more of such all-inclusive and well-designed studies with large sample sizes involving integrated advanced metagenomic, metatranscriptomic, metaproteonomic and metabolomic approaches needs to be conceptualized to study the compositional and functional aspects of the gut microbiome in malnourished children to substantiate the findings of the current study. Moreover, in-vivo and well-designed human clinical studies are also necessary for designing any targeted microbial therapies to prevent Severe Acute malnutrition in children in the near future.

Supplementary Information The online version contains supplementary material available at https://doi.org/10.1007/s00394-021-02571-7.

Acknowledgements We sincerely acknowledge ICAR-National Dairy Research Institute [NDRI], Deemed University, Karnal, India for providing necessary research facility for conducting research work. Indian Council of Agricultural Research [ICAR] for providing Junior Research Fellowship (JRF). Post Graduate Institute of Medical Education and Research (PGIMER), Chandigarh, India for assisting in sample collection.

Funding No funding was gained to support this project.

\section{Declarations}

Conflict of interest The authors declare that there is no conflict of interest.

Ethical approval The recruitment of volunteers, sample collection, and other study-related procedures were carried out by following the guidelines and protocols approved by the Institute Ethics Committee of PGIMER, Chandigarh, India.

Consent to participate Written informed consent was obtained from all subjects prior to any study-related procedures.

\section{References}

1. Keeley B, Little C, Zuehlke E (2019) The state of the world's children 2019: children. Food and Nutrition-Growing Well in a Changing World, UNICEF

2. Gupta SS, Mohammed MH, Ghosh TS, Kanungo S, Nair GB, Mande SS (2011) Metagenome of the gut of a malnourished child. Gut pathogens 3(1):7

3. Monira S, Nakamura S, Gotoh K, Izutsu K, Watanabe H, Alam NH, Endtz HP, Cravioto A, Ali S, Nakaya T (2011) Gut microbiota of healthy and malnourished children in Bangladesh. Front Microbiol 2:228

4. Subramanian S, Huq S, Yatsunenko T, Haque R, Mahfuz M, Alam MA, Benezra A, DeStefano J, Meier MF, Muegge BD (2014)
Persistent gut microbiota immaturity in malnourished Bangladeshi children. Nature 510(7505):417-4211

5. Dinh DM, Ramadass B, Kattula D, Sarkar R, Braunstein P, Tai A, Wanke CA, Hassoun S, Kane AV, Naumova EN (2016) Longitudinal analysis of the intestinal microbiota in persistently stunted young children in South India. PLoS ONE 11(5):e0155405

6. Méndez-Salazar EO, Ortiz-López MG, MdlÁ G-S, PalaciosGonzález B, Menjivar M (2018) Altered gut microbiota and compositional changes in Firmicutes and Proteobacteria in Mexican undernourished and obese children. Front Microbiol 9:2494

7. Alou MT, Bachar D, Levasseur A, Brah S, Alhousseini D, Sokhna C, Diallo A, Wieringa F, Million M, Raoult D (2019) Gut microbiota alteration is characterized by a proteobacteria and fusobacteria bloom in kwashiorkor and a bacteroidetes paucity in marasmus. Sci Rep 9(1):1-13

8. Wilson M (2005) Microbial inhabitants of humans: their ecology and role in health and disease. Cambridge University Press

9. Faith JJ, Guruge JL, Charbonneau M, Subramanian S, Seedorf H, Goodman AL, Clemente JC, Knight R, Heath AC, Leibel RL (2013) The long-term stability of the human gut microbiota. Science 341(6141): 1237439

10. Fan Y, Pedersen O (2020) Gut microbiota in human metabolic health and disease. Nat Rev Microbiol 19:1-17

11. Rakoff-Nahoum S, Paglino J, Eslami-Varzaneh F, Edberg S, Medzhitov R (2004) Recognition of commensal microflora by toll-like receptors is required for intestinal homeostasis. Cell 118(2):229-241

12. Kau AL, Ahern PP, Griffin NW, Goodman AL, Gordon JI (2011) Human nutrition, the gut microbiome and the immune system. Nature 474(7351):327-336

13. Evans JM, Morris LS, Marchesi JR (2013) The gut microbiome: the role of a virtual organ in the endocrinology of the host. J Endocrinol 218(3):R37-472

14. Kau AL, Planer JD, Liu J, Rao S, Yatsunenko T, Trehan I, Manary MJ, Liu T-C, Stappenbeck TS, Maleta KM (2015) Functional characterization of IgA-targeted bacterial taxa from undernourished Malawian children that produce diet-dependent enteropathy. Science Translat Med 7(276):276ra224-276ra224

15. Million M, Alou MT, Khelaifia S, Bachar D, Lagier J-C, Dione N, Brah S, Hugon P, Lombard V, Armougom F (2016) Increased gut redox and depletion of anaerobic and methanogenic prokaryotes in severe acute malnutrition. Sci Rep 6(1):1-113

16. Castro-Mejía JL, O’Ferrall S, Krych Ł, O’Mahony E, Namusoke H, Lanyero B, Kot W, Nabukeera-Barungi N, Michaelsen KF, Mølgaard C (2020) Restitution of gut microbiota in Ugandan children administered with probiotics (Lactobacillus rhamnosus GG and Bifidobacterium animalis subsp. lactis BB-12) during treatment for severe acute malnutrition. Gut Microbes 11:1-13

17. Kara S, Volkan B, Erten I (2019) Lactobacillus rhamnosus GG can protect malnourished children. Beneficial microbes 10(3):237-244

18. Tidjani Alou M, Million M, Traore SI, Mouelhi D, Khelaifia S, Bachar D, Caputo A, Delerce J, Brah S, Alhousseini D (2017) Gut bacteria missing in severe acute malnutrition, can we identify potential probiotics by culturomics? Front Microbiol 8:8994

19. Guerrant RL, Leite AM, Pinkerton R, Medeiros PH, Cavalcante PA, DeBoer M, Kosek M, Duggan C, Gewirtz A, Kagan JC (2016) Biomarkers of environmental enteropathy, inflammation, stunting, and impaired growth in children in northeast Brazil. PLoS ONE 11(9):e0158772

20. Claassen S, du Toit E, Kaba M, Moodley C, Zar HJ, Nicol MP (2013) A comparison of the efficiency of five different commercial DNA extraction kits for extraction of DNA from faecal samples. J Microbiol Methods 94(2):103-110 
21. Drake M, Small CL, Spence KD, Swanson BG (1996) Rapid detection and identification of Lactobacillus spp. in dairy products by using the polymerase chain reaction. J Food Prot 59(10):1031-1036

22. Lüdemann H, Conrad R (2000) Molecular retrieval of large 16S rRNA gene fragments from an Italian rice paddy soil affiliated with the class Actinobacteria. Syst Appl Microbiol 23(4):582-584

23. Dubernet S, Desmasures N, Guéguen M (2002) A PCR-based method for identification of lactobacilli at the genus level. FEMS Microbiol Lett 214(2):271-275

24. Huijsdens XW, Linskens RK, Mak M, Meuwissen SG, Vandenbroucke-Grauls CM, Savelkoul PH (2002) Quantification of bacteria adherent to gastrointestinal mucosa by real-time PCR. J Clin Microbiol 40(12):4423-4427

25. Rinttilä T, Kassinen A, Malinen E, Krogius L, Palva A (2004) Development of an extensive set of $16 \mathrm{~S}$ rDNA-targeted primers for quantification of pathogenic and indigenous bacteria in faecal samples by real-time PCR. J Appl Microbiol 97(6):1166-1177

26. Guo X, Xia X, Tang R, Zhou J, Zhao H, Wang K (2008) Development of a real-time PCR method for Firmicutes and Bacteroidetes in faeces and its application to quantify intestinal population of obese and lean pigs. Lett Appl Microbiol 47(5):367-373

27. Mühling M, Woolven-Allen J, Murrell JC, Joint I (2008) Improved group-specific PCR primers for denaturing gradient gel electrophoresis analysis of the genetic diversity of complex microbial communities. ISME J 2(4):379-392

28. Haakensen M, Dobson C, Deneer H, Ziola B (2008) Real-time PCR detection of bacteria belonging to the Firmicutes Phylum. Int J Food Microbiol 125(3):236-241

29. Balamurugan R, Rajendiran E, George S, Samuel GV, Ramakrishna BS (2008) Real-time polymerase chain reaction quantification of specific butyrate-producing bacteria, Desulfovibrio and Enterococcus faecalis in the feces of patients with colorectal cancer. J Gastroenterol Hepatol 23(81):1298-1303

30. Larsen N, Vogensen FK, Van Den Berg FW, Nielsen DS, Andreasen AS, Pedersen BK, Al-Soud WA, Sørensen SJ, Hansen LH, Jakobsen M (2010) Gut microbiota in human adults with type 2 diabetes differs from non-diabetic adults. PLoS ONE 5(2):e9085

31. Dong D, Liu W, Li H, Wang Y, Li X, Zou D, Yang Z, Huang S, Zhou D, Huang L (2015) Survey and rapid detection of Klebsiella pneumoniae in clinical samples targeting the rcsA gene in Beijing, China. Front Microbiol 6:519

32. Sambrook H (1989) Molecular cloning a laboratory manual. Cold Spring Harbor, NY

33. Xu Z, Knight R (2015) Dietary effects on human gut microbiome diversity. Br J Nutr 113(S1):S1-S5

34. Preidis GA, Ajami NJ, Wong MC, Bessard BC, Conner ME, Petrosino JF (2015) Composition and function of the undernourished neonatal mouse intestinal microbiome. J Nutr Biochem 26(10):1050-1057
35. Jain N, Walker WA (2015) Diet and host-microbial crosstalk in postnatal intestinal immune homeostasis. Nat Rev Gastroenterol Hepatol 12(1):14

36. Silva MJB, Carneiro MBH, dos Anjos PB, Pereira Silva D, Lopes MEdM, dos Santos LM (2015) The multifaceted role of commensal microbiota in homeostasis and gastrointestinal diseases. $\mathrm{J}$ Immunol Res 2015:321241

37. Hollister EB, Gao C, Versalovic J (2014) Compositional and functional features of the gastrointestinal microbiome and their effects on human health. Gastroenterology 146(6):1449-1458

38. Oriá RB, Murray-Kolb LE, Scharf RJ, Pendergast LL, Lang DR, Kolling GL, Guerrant RL (2016) Early-life enteric infections: relation between chronic systemic inflammation and poor cognition in children. Nutr Rev 74(6):374-386

39. Thomas F, Hehemann J-H, Rebuffet E, Czjzek M, Michel G (2011) Environmental and gut bacteroidetes: the food connection. Front Microbiol 2:93

40. Ghosh TS, Gupta SS, Bhattacharya T, Yadav D, Barik A, Chowdhury A, Das B, Mande SS, Nair GB (2014) Gut microbiomes of Indian children of varying nutritional status. PLoS ONE 9(4):e95547

41. Shin N-R, Whon TW, Bae J-W (2015) Proteobacteria: microbial signature of dysbiosis in gut microbiota. Trends Biotechnol 33(9):496-503

42. Hill CJ, Lynch DB, Murphy K, Ulaszewska M, Jeffery IB, O'Shea CA, Watkins C, Dempsey E, Mattivi F, Tuohy K (2017) Evolution of gut microbiota composition from birth to 24 weeks in the INFANTMET cohort. Microbiome 5(1):4

43. Xiao Y, Zhao J, Zhang H, Zhai Q, Chen W (2020) Mining Lactobacillus and Bifidobacterium for organisms with long-term gut colonization potential. Clin Nutr 39(5):1315-1323

44. Leimbach A, Hacker J, Dobrindt U (2013) E. coli as an allrounder: the thin line between commensalism and pathogenicity. Between pathogenicity and commensalism. Springer, pp 3-32

45. Chisti MJ, Tebruegge M, La Vincente S, Graham SM, Duke T (2009) Pneumonia in severely malnourished children in developing countries-mortality risk, aetiology and validity of WHO clinical signs: a systematic review. Tropical Med Int Health 14(10):1173-1189

46. Gurung M, Li Z, You H, Rodrigues R, Jump DB, Morgun A, Shulzhenko N (2020) Role of gut microbiota in type 2 diabetes pathophysiology. EBioMedicine 51:102590 